based remote sensing offers some opportunities to track biodiversity, our knowledge of species will only advance if we develop capacity for in situ monitoring, especially in biodiversity-rich countries (Stephenson et al., 2017, Frontiers in Ecology and the Environment, 15, 124-125).

In 2016 the IUCN Species Survival Commission (SSC) formed the Species Monitoring Specialist Group (https:// www.speciesmonitoring.org) to address the ongoing challenges with biodiversity monitoring. The Group's mission is to enhance biodiversity conservation by improving the availability and use of data on species populations, their habitats, and threats. Our objectives focus on improving the accessibility of appropriate tools and methods to fill data gaps, building capacity for monitoring, enhancing data collection and use (especially for neglected taxa), and ensuring databases are inter-linked and meet users' needs. The Group's unique aim is to enhance the volume and quality of data feeding into the IUCN Red List of Threatened Species. Group members-drawn from a variety of disciplines with experience as data collectors and users across regions and taxa-work in close partnership with key data stakeholders, including relevant organizations (e.g. UN Environment World Conservation Monitoring Centre, UNEP-WCMC), networks (e.g. Group on Earth Observations Biodiversity Observation Network), partnerships (e.g. the new Specialist Group is a Data User Partner of the Biodiversity Indicators Partnership) and alliances (e.g. the Eye on Earth Alliance), as well as government agencies, NGOs, local communities, academic institutions and the private sector.

One of the Specialist Group's challenges is to prioritize its efforts, and early work is focusing on identifying data and capacity gaps. In November 2017 that work began with the start of a project funded by the Cambridge Conservation Initiative Collaborative Fund (http://www.cambridgeconserva tion.org/collaboration/global-audit-biodiversity-monitoring). The Specialist Group, Birdlife International, RSPB, the University of Cambridge, UNEP-WCMC and the Zoological Society of London are working with their network of partner organizations to conduct the first global audit of biodiversity monitoring. This will include identifying the main geographical and taxonomic gaps in biodiversity monitoring, compiling an inventory of schemes and the methods they use, and linking data collectors with data users. In parallel, the Group is reviewing data needs across the SSC taxonomic specialist groups by surveying members, and reviewing lessons learned on species monitoring from large project portfolios, such as the IUCN Save Our Species Programme. The Group is also planning more in-depth analyses of global databases, building on recent studies (e.g. McRae et al., 2017, PloS One, 12, e0169156). Complementary work underway includes the identification of sites in Africa, Asia and Latin America where partner agencies will help develop and test standardized monitoring methods and protocols to enhance collection of data on poorly-known species, including the application of the proposed IUCN Green List of Species (Akçakaya et al., 2018, Conservation Biology, https://doi.org/10.1111/cobi.13112). The Group's chair is also working with the IUCN Business and Biodiversity Programme to explore options for biodiversity monitoring frameworks for the private sector, building on collaborative work started with the energy company Enel (https://www. iucn.org/news/business-and-biodiversity/201710/iucn-andglobal-energy-company-enel-develop-biodiversity-bestpractices).

The initiation of the Species Monitoring Specialist Group will ensure that by 2019 IUCN and its commissions, members and partners, and the broader academic and conservation communities, will have a clearer idea of where biodiversity data are most needed. The aim then will be to focus the Group's attention and expertise on building capacities to fill identified gaps. Ultimately this will lead to an improvement in conservation planning, the enhanced monitoring of project impacts and outcomes, and improved results-based reporting by governments on global environmental goals such as the Aichi Targets and the Sustainable Development Goals.

P.J. STEPHENSON Chair, IUCN Species Survival Commission Species Monitoring Specialist Group, c/o Ecosystem Management Group, Department of Environmental Systems Science, ETH Zürich, Zürich, Switzerland.

E-mail stephensonpj@gmail.com

\section{Restoring nature with evidence}

A new website, http://www.restorationevidence.org, is gathering and summarizing scientific evidence for the effectiveness of interventions to restore degraded habitats. A subset of https://www.conservationevidence.com dealing only with restoration, the site is a collaboration between the Endangered Landscapes Programme and Conservation Evidence, making evidence freely available to all. This will help ensure funds are spent on interventions that are most likely to be effective in achieving a desired outcome, from restoring upland grazing pastures into blanket bog to converting ranches into tropical forests.

So far, the site covers the effectiveness of actions to restore peatland vegetation, shrublands and heathlands, and forests, and evidence for the impacts of habitat restoration on several animal taxa. Work is underway to add the impacts of restoration techniques for grasslands, wetlands and benthic marine habitats, and for more animal taxa. The goal is eventually to cover all interventions that could be used to restore all habitats globally, and to update these regularly to include the latest evidence.

Restoration Evidence makes the evidence for the effects of restoration interventions accessible and digestible, so that 
planners and managers can rapidly obtain an overview of what has and hasn't worked. For each intervention, such as 'rewet peat' or 'use fences to exclude livestock from shrublands', each article or publicly available report that has tested the intervention is summarized in a paragraph written in a standardized manner, making the methods and results as clear as possible. The overall findings from these studies are summarized as a set of key messages, giving a rapid overview of the effects of a given intervention. Experts score each intervention in terms of how effective the intervention seems to be (based on available evidence), the level of certainty in the current evidence, and potential harm that might arise to the target taxa or habitat from this intervention. The methods used for finding and synthesizing the evidence can be found on the Conservation Evidence website.

Habitat restoration is increasingly recognized as having an important role to play in conserving biodiversity, mitigating climate change and improving well-being, and this is reflected in the creation of international targets for restoring habitats. The Convention on Biological Diversity aims to restore $15 \%$ of degraded ecosystems by 2020 , and the Bonn Challenge sets out to restore 350 million ha of deforested and degraded lands by 2030. Regional initiatives contributing to the Bonn Challenge have also been established. Initiative $20 \times 20$ aims to bring 20 million ha in Latin America and the Caribbean into restoration by 2020 , and AFR1oo aims to start restoration on 100 million ha across Africa by 2030. The UK's 25 year Environment Strategy has a target to create or restore 500,000 ha of wildlife-rich habitat outside protected sites. Initiatives such as these will have maximum impact if they use evidence on which interventions are most likely to be most effective. Having the answers to questions such as 'how should we remove invasives' and 'when should we plant trees and when should we sow seeds instead' is critical to the effective use of resources.

So what does work in restoration? For each ecosystem there are interventions that seem to be effective, although the exact effects are likely to vary with local conditions. In peatlands, rewetting peat and scattering mosses on the peat surface were effective in many studies. For shrublands and heathlands, reducing livestock numbers or density seems to be important. For forests, preparing the ground before planting trees and then thinning the planted trees was effective.

One of the most important findings from the creation of RestorationEvidence.org has been that for each habitat there are relatively few high quality studies testing interventions, meaning that many actions are classified as 'unknown effectiveness'. If every restoration project included just one experimental component, in which the effects of an intervention were tested, monitored and published, we would know a lot more about the best ways to restore ecosystems.

ClatRE WORDLEY Conservation Evidence, The University of Cambridge,UK.E-mail cfw41@cam.ac.uk
NANCY OCKENDON Conservation Evidence, The University of Cambridge, and The Endangered Landscapes Programme, Cambridge, UK

David Thomas The Endangered Landscapes Programme, Cambridge, UK

\section{The Whitley Awards 2018}

The 25th Anniversary Whitley Awards Ceremony was held on 25 April 2018 at the Royal Geographical Society in London. The flagship event of UK-based charity, the Whitley Fund for Nature (WFN), was hosted by wildlife presenter and WFN ambassador Kate Humble. The evening saw six conservation leaders from six different countries receive Whitley Awards worth GBP 40,000 each in project funding over 1 year in support of their work.

In addition, the prestigious Whitley Gold Award worth GBP 60,000 was presented to 2010 Whitley Award winner Pablo Borboroglu of the Global Penguin Society, Argentina, for his work to conserve penguins and their habitat throughout the Southern Hemisphere. Over 500 people attended the event, where the charity's patron, HRH The Princess Royal, presented the Awards.

The 2018 Whitley Award Winners are: Dominique Bikaba, Democratic Republic of the Congo (Ensuring the survival of DRC's eastern lowland gorillas); Kerstin Forsberg, Peru (Majestic giants: safe passage for manta rays in Peru and Ecuador); Olivier Nsengimana, Rwanda (Conserving Rwanda's emblematic grey crowned crane): Shahriar Caesar Rahman, Bangladesh (Tortoises in trouble: community conservation of Asia's largest tortoise); Munir Virani, Kenya (Game of poisons: a strategy to save Kenya's threatened vultures); Anjali Chandraraj Watson, Sri Lanka (Leopards as a flagship for wildlife corridors).

Over its 25 years, the charity has given almost GBP 15 million in conservation funding to more than 190 conservation leaders in over 80 countries. The Whitley Award was one of the first awards to be given in recognition of effective conservation leadership in the Global South. Twenty-five years on, the need for recognition of grassroots leadership has never been stronger. The Fund supports emergent conservation leaders who are nationals of the countries where they work, making them best placed to lead change and articulate solutions. Through them WFN supports work rooted in community involvement that is pragmatic, science-based, and has lasting impact.

Change doesn't happen overnight and WFN's commitment to conservation leaders is long term. Winners benefit from membership of a global network of Whitley Award alumni, and are able to apply for WFN Continuation Funding to scale up their work and bring about positive 